\title{
LA MORALE DE LA VÉRITÉ
}

Qu'est-ce que c'est que la vérité chez Sartre? Même sans avoir pris connaissance de Vérité et existence, nous aurions pu reconstruire une épistémologie existentialiste. Il aurait été suffisant de combler les lacunes que nous avait laissé Sartre parce que nous savions déjà ce que cette thérie devrait comprendre: une ontologie de la vérité en termes du jeu entre le pour-soi et l'ensoi; une insistance sur le rôle central de l'action humaine; les efforts faits par Sartre pour lier sa pensée de l'époque de l'avant-guerre et de L'Etre et le Néant avec sa politisation et sa radicalisation subséquente, voire sa préoccupation pratique et théorique croissante de l'Histoire. Dans Vérité et existence, rédigé en 1947-1948 et édité soigneusement par Arlette Elkaìm-Sartre, Sartre ne déçoit pas: parmi les thèmes clefs, ceux-là justement ont chacun une élaboration très développée. Mais, au-delà de nos anticipations qui peuvent quelquefois ne pas être fondées, Sartre parle moins de la vérité qu'il ne parle de l'ignorance.

En fait, la plupart du texte porte sur les structures et les comportements humains négatifs envers la vérité; et la plus grande partie du texte (peut-être 80 pour cent) traite de la question de l'ignorance. Cela veut dire que l'ignorance, bien qu'elle soit fondée sur la condition ontologique originelle de l'ignorance (comme l'est tout savoir), est une action entreprise par choix. Il est encore mieux de dire que c'est une action visant à éviter ou à cacher un aspect de la vérité, ou encore, que c'est une forme de mauvaise foi. Ainsi, au centre de Vérité et existence se trouvent des descriptions de l'ignorance en tant qu'acte; des analyses des conduites cherchant à éviter la vérité; et les raisons pour lesquelles elles évitent la vérité. C'est la raison pour laquelle je voudrais parler d'une morale de la vérité chez Sartre. ${ }^{1}$ La plupart de mes analyses porteront sur le rôle central de la volonté, donc de la morale, dans Vérité et existence. Puis je ferai quelques remarques critiques sur la morale de la vérité chez Sartre.

\footnotetext{
1"Aussi n'y a-t-il pas une vérité de la conscience (de) soi mais une morale, en ce sens qu'elle est choix et existence qui se donne des règles dans et par son existence pour exister" (p. 93).
} 
Le point de départ du texte est la vérité comme dévoilement progressif de l'être-en-soi par le pour-soi. La vision d'un individu est donné à l'autre, qui la fait sienne en la dépassant pour réaliser ses propres fins. Donc il s'agit de l'histoire prise dans un double sens: 1) "La vérité se temporalise, c'est-à-dire qu'elle apparaît selon les catégories d'avant et d'après" (p. 45); en d'autres termes, l'être se dévoile "d travers toute l'hisoire humaine" (p. 25). 2) Sartre nous dit que "le jugement est un phénomène interindividuel" (22). Bien que ce soit une chose que de voir et une autre que de juger, la vue et le jugement s'enchevêtrent. A cause de cela, "je ne vois souvent qu'en indiquant. Ainsi l'homme voit pour l'autre ou voit le déjà vu" (p. 22). Ce qui revient à dire que la vérité comprend l'intersubjectivité.

Ce sont tous des thémes nouveaux et importants, mais pour bien les illustrer, Sartre aurait da mener cette étude jusqu'aux limites de sa pensée. Mais en 1948 c'était encore trop tót et il a préféré continuer son projet d'éclairer en tant qu'action tous les domaines humains. Ainsi il y a une continuité essentielle entre Vérité et existence et les analyses de l'avant-guerre, comme par exemple L'Imaginaire et Esquisse d'une théorie des émotions, ainsi que L'Etre et le Néant.

Après avoir introduit l'idée de l'ignorance, Sartre essaie de démontrer les liens internes de l'ignorance avec le savoir, ces derniers portant sur le fait que rien ne m'est donné par le monde et tout est saisi par moi. Comme l'indique déjà L'Etre et le Néant, la catégorie de l'action vise ce qui $n$ 'est pas-soit un état futur de l'Etre, soit "ce que je voudrais qu'il soit" (p. 47)-d'où l'anticipation comme thème central de l'action dévoilante: l'anticipation "précède [chaque] vision et la construit. . . Celle-ci sert alors de mesure et de schéma directeur de la vision" (pp. 48-49). En anticipant, je peux organiser l'en-soi sous ma vue: "je crée ce qui est" (p. 49). Ne prenons pas la phrase "ce qui est" dans le sens idéaliste, parce qu'il s'agit ici d'un en-soi indépendant qui se donne toujours comme être-déjà, comme le dévoilement et le surgissement du Poursoi. $^{2}$

\footnotetext{
${ }^{2}$ Il faut remarquer que ces textes renversent effectivement l'argument de L'Imagination et de L'Imaginaire, qui parient de la perception comme passive et mettent l'accent sur la spontanété de l'imagination.
} 
Qu'est-ce donc que l'erreur? Une vérification négative de nos anticipations: l'arbre que $\mathrm{j}$ 'attendais n'est vraiment pas un arbre, mais quelque chose d'autre. "Dans l'erreur, en effet, l'Etre n'est pas ce qu'on en dit . . ." (54). Ainsi la vérité comporte toujours un risque d'erreur. D'ailleurs elle est toujours en train d'être vérifiée par la pratique, qui ne cesse jamais d'utiliser ses objets, de les anticiper-donc de risquer, de projeter. L'erreur "est nécessaire à la vérité parce qu'elle rend la vérité possible" (p. 57).

On pourrait dire avec Heidegger: sans libérté, pas de vérité. Ainsi, au-delà de toutes les conséquences décrites dans L'Etre et le Néant, le surgissement du Pour-soi entraîne de nouvelles possibilités conjointes: l'ignorance et le savoir, l'erreur et la vérité. "L'ignorance conditionne le savoir et se définit par lui, " dit Sartre, "c'est-à-dire à la fois comme possibilité de savoir ou possibilité de demeurer ignorance" (p. 60). Les différentes possibilités vont ensemble, comme Heidegger l'a décrit dans $L$ 'Essence de la Vérité. Mais Sartre prend sa distance de Heidegger en mettant l'accent sur le choix individuel. Ainsi l'ignorance, qui est toujours entrainée par le savoir, est aussi un comportement libre; en tant que tel, elle dépend du vouloir. Comme "je dois décider la vérité, le vouloir, donc je peux ne pas le vouloir." Sartre continue: "La condition pour que la vérité soit, c'est la pérpétuelle possibilité de la refuser" (59). Bien qu'ici il semble parler comme Heidegger, ce qui concerne Sartre n'est ni le refus historique ni le refus ontologique de l'être primordial mais le refus de l'individu face à ses responsabilités.

\section{III}

Voilà la base théorique sur laquelle Sartre va décrire le choix de "laisser voilé" l'être. Au début de l'analyse ontologique de ce texte, Sartre utilise un ton qui frappe ceux qui se rappellent du ton conflictuel et négatif des relations du Pour-soi et de l'En-soi dans L'Etre et le Néant. Comme l'a déjà rémarqué Juliette Simont ${ }^{3}$, il décrit maintenant les rapports entre le Pour-soi et l'En-soi comme étant mutuellement affirmatifs plutôt que comme des rapports antagonistes ou de mauvaise foi. En effet le comportement vérifiant "suppose nécessairement un goût d'être" (p. 60), et ce gout cherche à ne rien cacher, à

\footnotetext{
${ }^{3}$ Voir par exemple l'article précédent dans cette revue.
} 
ne rien éviter. Je commence à dévoiler l'être, je n'existe que pour le faire apparaître.

Sartre va encore plus loin et dit que: "Cette irritante et voluptueuse proximité sans distance du Pour-soi à ce qui n'est pas soi est précisément la jouissance" (63). Cette remarque est une élaboration d'un texte de $Q u$ 'est-ce que la littérature?, où la création d'un objet esthetique donne de la joie et de la jouissance: dans un texte de Qu'est-ce que la littérature, qui est capital pour l'analyse de Vérité et existence, Sartre parle "d'un calme souverain des émotions esthétiques," et également d'une "harmonie rigoureuse entre la subjectivité et l'objectivité. ${ }^{n}$

Cela veut dire que le monde-l'En-soi en train d'être dévoilé-ne m'apparaît plus en ce moment en tant qu'obstacle ou en tant que menace, ni comme outil ni comme "la distance infinie qui nous sépare de nous-mêmes. " 5 La tension du Pour-soi-En-soi reste en suspens, l'homme n'est plus une passion inutile: l'En-soi apparaît au Pour-soi comme "un monde qui est à la fois son monde et le monde extérieur. ${ }^{n 6}$ Par conséquent, selon Vérité et existence, on peut "assumer le monde comme si on l'avait cré, en prendre son parti, prendre le parti de l'Etre (parti pris des choses), se faire responsable du monde comme s'il était notre création" (p. 63). ${ }^{7}$

Ensuite Sartre parle d'aimer le vrai, de faire face aux faits, même ceux qui gênent, de "préférer L'Etre à tout, même sous une forme catastrophique, simplement parce qu'il est" (p. 63). Il en donne des raisons pratiques et ontologiques. Pratiquement, c'est absurde de vouloir ignorer la vérité: nos projets, quelqu'ils soient, s'organisent autour d'un certain dévoilement de l'être. Ainsi, par exemple, le mariage est basé sur la fidélité et sa vérification est

\footnotetext{
4"Qu'est-ce que la littérature?" Situations, Il (Paris, 1948), p. 108.

${ }^{5}$ Ibid.

${ }^{6}$ Ibid., p. 109.
}

'Sartre a déjà prévu ceci en parlant de l'art, du jeu, et du savoir. Dans chaque cas il constate une réussite ontologique, c'est dire l'expérience/création d'un objet extérieur qui est à la fois d moi. Mais chaque fois ce qui intéresse Sartre surtout c'est l'échec éventuel et nécessaire. Voir $L$ 'Eure et le Néant (Paris, 1943), pp. 665-82. 
toujours en cours; elle est une partie essentielle du projet mutuel de se marier. En effet, il serait malhabile de choisir l'ignorance, la forme de comportement décrite dans La Coup enchantée de La Fontaine, qui visait à laisser se voiler les faits et les gestes de ma femme quelqu'en soient leur nature. Tốt ou tard, la réalité me frappera et $j$ 'en serai la victime, parce qu'en choisissant l'ignorance, je préfere, en tant que thème régulateur de ma vie, le hasard a l'action révélatrice.

D'ailleurs, cette dénégation de mon projet entraine des conséquences ontologiques. Dans l'ignorance, je nie que la tâche de la réalité-humaine soit le dévoilement de l'être. Donc, je contredis mon surgissement même dans le monde. D'ailleurs en l'ignorant, j'essaie de donner un moindre être à l'être nonrévélé. Mais cela est impossible; en réalité, je ne peux que diminuer mes rapports avec le monde, comme Sartre les a esquissés dans ses oeuvres psychologiques. "L'ignorance," dit-il dans Vérité et existence, "est décision de laisser s'effondrer l'Etre" (p. 68). J'essaie de m'en laver les mains.

Une femme tuberculeuse, Madame T., choisit de ne pas aller voir de médecin parce qu'elle veut minimiser le danger et l'ignorer bien qu'elle en soit consciente. Si on lui confirmait l'existence de sa maladie, cela lui donnerait de nouvelles responsabilités; parce que la vérification est la création de l'être, celui qui vérifie prend alors la responsabilité de cet aspect de la réalité, et en devient complice. "T. refuse de prendre la responsabilité de faire accéder au monde humain ce qui n'a que l'existence larvaire d'un monde souterrain et nocturne. Elle refuse de se choisir tuberculeuse et de créer librement la tuberculose" (p. 70). Cela ne change rien à la toux, au crachement de sang, à la fièvre, mais ces phénomènes sont vécus par eux-mêmes, chacun isolé de l'autre, sans être vus en tant que tels. Même quand la tuberculose la tuera, T. sera restée passive, ignorante, sans responsabilité, victimisée par le hasard. En même temps elle fuit sa liberté: "la peur de la vérité est peur de la liberté" (p. 70).

Bien sûr, on doit choisir d'ignorer une partie de la réalité pour en savoir une autre parce que tout savoir commence par l'ignorance et reste entouré de l'ignorance. Mais si T. s'absorbait à poursuivre n'importe quelle activité, par exemple à vérifier son talent dramatique, et par conséquent n'avait pas le temps de rendre visite au médecin, cela serait une distraction hystérique. La conscience joue à supprimer la maladie jusqu'à la mort afin de pouvoir oublier ce dont on a peur; en réalité, en supprimant la conscience de la maladie, on s'approche de 
l'état qu'on redoute le plus. Ainsi l'ignorant "prend le point de vue de la mort par peur de la mort. .." (p. 75).

Tout ceci relève donc de la mauvaise foi: $T$. prétend être controlée par le destin et la fatalité pour éviter ses responsabilités face à cette tuberculose. En revanche, la vérité supprimée devient la préoccupation totale de l'ignorant: la tuberculose est "le thème organisateur de ses événements intimes; " même si "ce thème a perpétuellement un moindre être que l'Etre, il est noème de mon inquiètude, signification d'un signe, corrélatif d'un acte imageant. . ." (p. 78). Dans ce jeu de plus en plus complexe, je nie ma transcendance, je me mens, je me rends impuissant: "pour conférer un moindre être à ce qui me menace, je me confere une moindre liberte" (p. 81).

Pour expliquer pourquoi on s'effraie devant l'Etre, Sartre retourne aux rapports du Pour-soi et de l'En-soi esquissés dans L'Etre et le Néant, et met l'accent sur le fait que l'Etre est effrayant. Il est, après tout, refus de mon existence; il est de trop et il fait que, moi aussi, je me sens de trop. ${ }^{8}$ L'Etre est caractérisé par l'impénétrabilité absolue, le mystère, le froid glacial, l'irréparabilité, l'impossibilité d'être récupéré. Il me fait face comme un ennemi, comme une chose exigeante, comme condition non modifiable, comme impitoyable-tout est entrainé par son caractère d'étant-déja. Je ne peux jamais changer ce qu'il est déjà; ma liberté se limite à cette perpétuelle ambiguité de me rendre responsable de ce que je n'ai ni cré ni voulu. Mais pour exister la conscience doit révéler cet être-ci et pas un autre-c'est sa condition d'existence dans un monde d'exigences données et objectives, c'est-à-dire c'est le choix sans choix. En affirmant donc la tension ontologique originelle, Sartre suggère en effet les raisons pour lesquelles la conscience veut se révolter contre les nécessités de son surgissement même.

Le surgissement a lieu toujours en tant que projet, et je me perds dans le monde de moyens-impératifs, écrasé par la nécessité d'accumuler des moyens pour réaliser mes fins. L'ignorance est une révolte qui vise à refuser ce travail perpétuel du monde des moyens; mais puisque la libérté est travail, l'ignorance est done le refus de la liberté même. En choisissant l'ignorance je veux créer

\footnotetext{
${ }^{8}$ A l'inverse de la page Sartre ajoute une note: ce refus c'est "le premier thème de la vie en société; c'est le sens même du travail." Celte idée eat suivie de trois pensées numérotées dont voici la dernière: "Antagonisme des hommes et rareté des biens. Ambivalence de la vie sociale. L'autre est celui qui partage avec moi et qui me vole ma nourriture" (p. 84).
} 
sans travailler, c'est-à-dire faire de mon désir "le moteur universel de création." J'essaie de supprimer l'indépendance de l'être et de le reconstruire comme extension de mon esprit, voire, n'avoir plus de responsabilités sauf vis-à-vis de moi-même. Donc l'ignorance cherche à réinstaller l'absolu-sujet hégélien comme pure conscience produisant son propre monde. ${ }^{9}$

Dans une série de réflections brèves et provocatrices, Sartre va plus loin dans son analyse de la fuite devant l'acte de vérifier et décrit les trois derniers modes de comportement vis-à-vis de la vérité, à savoir l'innocence, la contemplation, et le savoir abstrait. Tout comme l'ignorance, l'innocence revient à choisir de ne pas être responsable du monde. Mais l'innocent, protégé des laideurs du monde (peut-être en tant que symbole d'une enfance perdue dont nous avons la nostalgie), devient pour nous un sage de qui l'ignorance vaut mieux que le savoir d'une société scientifique et technique.

L'histoire d'Adam et Eve nous donne un autre aspect de l'ignorance: la contemplation passive d'une vérité "déjà constituée avant nous" (103). Cette dernière rompt le rapport qui existe entre la vérité et notre liberté et notre action de dévoilement. Ici, nous n'avons pas affaire à la vérification: la vérité nous est donnée par "l'existant qualifié pour faire exister le Vrai" (p. 103), cela veut dire Dieu, ou peut-être encore Hitler ou Staline; ou bien des autorités scientifiques qui nous "donnent" des vérités menaçantes comme l'énergie atomique. En en faisant des sujets absolus et "dévoileurs," en les critiquant pour les vérités qu'ils nous obligent à accepter, en oubliant que toute vérification est toujours en cours,

\footnotetext{
9 En parlant du schizophrène, Sartre fait référence implicite à L'Imaginaire: "Il n'est pas vrai que le schizophrène préfêre le rêve parce qu'il y apparaît comme millionnaire, empéreur, etc. Il prérere le monde du rêve parce que l'être n'y est que dans l'exacte mesure où il est dévoilé: il prêfere la pauvreté de l'être, et parce que l'être est un moindre âtre, qui se résorbe aussitót en subjectivité, et parce que entre l'être désiré et l'être désirant il n'y a aucun intermédiaire" (p. 99).

Comparer $L$ 'Imaginaire: "DEs lors nous pouvons bien penser qu'on devra ranger les individus en deux grandes catégories, suivant qu'ils préferent mener une vie imaginaire ou une vie réelle. Mais il faut bien comprendre ce que signifie la préférence de l'imaginaire. Il ne s'agit nullement de préférer seulement des objets à d'autres. Il ne faudrait pas croire, par exemple, que le schizophrène, et, d'une façon générale, les réveurs morbides tentent de substituer au contenu réel de leur vie un contenu irrél plus séduisant, plus brillant, et qu'ils chenchent a oublier le caractère irréel de leurs images en se conduisant vis-d̀-vis d'elles comme s'il s'agissait d'objets actuellement et réellement présents. Préférer l'imaginaire ce n'est pas seulement préférer une richesse, une beauté, un luxe en image à la médiocrité présente malgré leur caractère irréel. C'est adopter aussi des sentiments et une conduite 'imaginaires', d cause de leur caractère imaginaire" (Paris, 1940), p. 189.
} 
nous nous cachons notre propre résponsabilité de faire en sorte que la vérité soit.

Ces formes de mauvaise foi peuvent nous mener a l'acte de savoir sans verifier, sans voir, sans dévoiler l'être par l'intuition. Il s'agit de raisonnements et de discours qui refusent à l'intuition "sa valeur révélante fondamentale" (p. 107). Ce genre de pensé ne pourrait exister que par le mitsein: "L'homme abstrait pense sur la pensée des autres, c'est-à-dire sur des révélations qu'il n'effectue pas" (p. 107). Il "Raisonne non parce qu'il ne voit pas mais pour ne pas voir" (p. 107). Voilà le dernier élément de la critique sartrienne de l'ignorance: dans le savoir abstrait on est absent, "par peur de l'En-soi."

Il reste une dernière étape à l'étude: selon Sartre certains aspects de la vérité restent nécessairement dans l'ombre, ce qu'il appelle la "face d'ombre." Donc chaque vérification est choix de vouloir se limiter, chaque savoir comporte sa finitude, et chaque action historique ne pourrait jamais se reconnaître dans l'objectivité qu'elle vise. Comme toutes les analyses dans Vérité et existence celles-ci sont à la fois brèves, nettes, et perspicaces. De toute façon, ce qui nous intéresse maintenant ce sont que les bases théoriques de toute l'entreprise épistémologique sartrienne. On peut dire que Sartre ne nous donne ni une description détaillée de la vérité ni une esquisse de la vérification. A vrai dire, Sartre ne nous les donne que par négation, quand il parle de l'ignorance. Vérité et existence est en effet une épistémologie de la face d'ombre. La moralisation qui anime tout ce texte est basée sur une théorie de la vérité qui reste, la plupart du temps, implicite et qui émerge furtivement de l'ombre. Cette vision peut nous mener aux problèmes irrésolus de l'entreprise épistémologique sartrienne.

"Quel est le critère de la vérité?" demande Sartre. "Aucun doute là-dessus: c'est l'Etre comme présence" (p. 110). Mais que veut-il dire par présence? Il dit: "Tout part de la vue et aboutit à la vue (intuition)" (p. 37). C'est la vision directe, donc individuelle, donc absolue, de l'être dévoilé: c'est mon expérience personnelle mais pas du tout "un épiphénomène non révélateur et purement subjectif" (p. 119). C'est plutôt le sujet qui éprouve l'objet de mon expérience directe de l'être-même si la vision globale comprend mon environnement, "un certain horizon de valeurs, de fins et de significations" (p. 116). 
La vérité ne se trouve pas dans l'énoncé; il ne s'agit pas d'une structure intellectuelle ou linguistique quelconque. Pour mieux la comprendre il faut situer cette individualisation de la vérité vis-à-vis d'autres approches épistémologiques possibles. On ne peut l'énoncer, ni la trouver dans une forme médiatisée, ni la construire avec des rapports intersubjectifs. Ainsi, la théorie sartrienne ressemble tantôt à un réalisme absolu-l'objet est là et attend son dévoilement. Tantôt elle ressemble à un intuitionnisme absolu -on voit l'objet directement et immédiament. Et tantôt elle ressemble à un subjectivisme absolu-l'objet est l'intuition de chacun de nous, en tant que conscience individuelle. C'est une épistémologie curieuse, mais elle a pour but de préserver un monde hors de la conscience et une.conscience libre et active, ainsi que des rapports entre les deux dans lesquels ni l'un ni l'autre n'est absorbé. Mais puisque la vérité est une vision directe de l'être, comment distinguer entre les aspects de ma vision qui sont à moi et ceux qui appartiennent à l'être même, c'est-à-dire entre le subjectif et l'objectif? Pour Sartre cette question classique n'est pas essentielle; de plus, sa théorie de l'intuition ne pose jamais le problème de la préparation d'une vision, comme nous voyons chez Descartes et dans la phénoménologie.

Afin de l'éclairer on devrait poser la question: si l'intuition comprend une vision directe de l'être, comment la communiquer aux autres? Même si, comme le dit Marx, telle vision peut être expliquée à partir de telle situation sociale, elle pourrait être "révélation absolue et absolument transmissible de l'Etre" (p. 120). Mais comment la transmettre? J'invite l'autre à voir ma vision personnelle, à la depasser en faisant d'elle sa vision également personnelle. "Je lui désigne l'objet et il le regarde. Il le regarde au bout de mon doigt. Mais dès lors, l'objet développe une dimension d'être qui m'échappe a priori." Lui, il voit cette fleur rouge à partir d'un "autre système de vérité" (p. 116). Ainsi ma vérité reçoit une limitation externe qui fait qu'elle n'est plus que ma vérité. Evidemment, la présence des autres n'ajoute rien à ma vision et en fait ils la limitent. En effet, comme nous venons de le voir, le mitsein est obstacle a la vision directe, car l'énoncé remplace la vision directe. ${ }^{10}$

Selon Sartre la vérité universelle existe (et en ceci les postmodernistes se distinguent de Sartre), mais elle n'est pas communiquée-on ne peut donner qu'une invitation: "le pur index d'une possibilité permanente et valable pour tous

\footnotetext{
${ }^{10}$ Sartre même dit que le mitsein est une structure tout à fait secondaire au "monde d'exigences objectives" (p. 94).
} 
de réaliser librement un certain dévoilement" (p. 120). Est-ce le même dévoilement pour tous? Pas du tout, parce que nous avons tous une histoire personnelle différente, etc., mais si nos perspectives sont différentes, nous dévoilons tous quand même le même être. " Nos visions, nos dévoilements sont vrais dans un sens absolu: c'est-à-dire que chacun de nous vit une expérience directe et immédiate de l'être (pp. 34-5). Comment le vérifier? Seulement par l'intuition, c'est-à-dire par la subjectivité qui veut voir la réalité. C'est la preuve la plus importante: elle comprend la bonne foi envers l'être, le choix de le voir, donc de vouloir voir, et de refuser l'ignorance. Et au-delà, aucune autre preuve n'est nécessaire, parce que la vérité reste axée sur l'intuition directe.

Comme nous le savons déjà, l'un des thèmes sartriens les plus importants est la mauvaise foi-vouloir cacher ou éviter la vérité. Ce qui importe dans la morale de la vérité sartrienne n'est jamais l'intelligence, ni les preuves rigoureuses, ni le raisonnement, parce qu'en fait, il n'est pas vraiment difficile de voir ce qui est. Ainsi la vérité n'exige aucune explication; sans l'ignorance qui la masque tout le monde peut la voir. Ce sont les points clefs de la thérie sartrienne de la vérité qui la distinguent en même temps de toutes les autres théries, y compris celle de Heidegger.

Cette thérie radicale peut nous aider à expliquer à la fois la grande vague sartrienne de l'après-guerre et l'absence d'épigones de Sartre aujourd'hui. Sartre a agi en accord avec ses paroles: dévoilant la réalité, attaquant tous ceux qu'il croyait coupable de mauvaise foi. Au-delà de la rhétorique et de l'hypocrisie bourgeoise ou communiste, Sartre a réussi à dévoiler des aspects capitaux de notre monde et à nous presenter des intuitions éblouissantes. Pas d'argumentation, pas de raisonnements: il s'agissait de l'évidence même et directe, dont nous trouvons la description chez Sartre. "Ou bien vous voyez ou bien vous êtes un salaud"-voilà la réplique abrupte concevable de Sartre à tout ceux qui l'ont contredit.

Sans doute j'exagére. mais ce que je voudrais souligner ce sont les liens entre un certain refus d'argumentation chez Sartre, la conception de la vérité en tant qu'intuition, la mauvaise foi comme thème capital, et la théorie de la

\footnotetext{
"Sartre l'explique bien en parlant dans Qu'est-ce que la liutrature? de la fonction de l'écrivain: son "action par dévoilement" (p. 73) nous montre notre monde pour que nous en assumions la responsabilité (p. 98).
} 
littérature engagée, ainsi que le style péremptoire de la pratique sartrienne morale et politique. Si, selon Sartre, l'ignorance part du vouloir, il ne vaut pas la peine d'être patient, de raisonner, de développer un argument, de convaincre; et, encore, si, selon Sartre, l'ignorance est un choix, elle est un comportement qu'on doit changer avant de réussir a voir la vérité. "Vous n'avez pas raison" (suivi par une explication) serait remplace chez Sartre par "Vous êtes de mauvaise foi."

Tout cela pourrait aider à expliquer l'absence frappante de sartriens après Sartre. Bien sûr, il nous a laissé des idées et des thèmes capitaux, des descriptions brillantes et puissantes, des oeuvres merveilleuses. En effet, on pourrait dire que dans un sens tout le monde a fini par adopter l'attitude de Sartre: l'idée de la liberté en tant que responsabilité, son idée, est devenue le fil conducteur de la conscience actuelle. Mais il existe de nos jours peu de personnes qui s'identifient suffisamment à Sartre afin qu'on puisse les appeller des "sartriens." Je pense que l'accent mis par Sartre sur l'intuition et sur le savoir et tant que vouloir en sont deux raisons. Sans méthode explicite, sans qu'il esquisse une objectivité communicable, l'intuitionnisme sartrien reste ancré dans les intuitions de quelqu'un, c'est-à-dire de Sartre lui-même. Alors, si certaines idées de Sartre ont vieilli, si d'autres ont été absorbées dans la conscience générale, ce qui est naturel, il faut se demander si la philosophie sartrienne aurait mieux survécu si Sartre avait développé une méthode, une approche de la vérification? En tout cas, ceci reste une tâche qui nous incombe: faire de cette discussion provocatrice et brillante de l'ignorance une partie d'une thérie plus complexe, moins moralisante, plus nuancée, moins simple, plus développée-mais qui continue à accentuer le rôle du vouloir et de l'action comme thème central.

Wayne State University

RONALD ARONSON 